\title{
Dietary factors in the aetiology of heart disease
}

\author{
IAN MaCDONALD \\ M.D., Ph.D., D.Sc. \\ Department of Physiology, \\ Guy's Hospital Medical School, London, S.E.1
}

CORONARY heart disease is probably a multifactorial disease, and it is not known for certain whether dietary factors are to be included in the list of predisposing causes, though evidence is accumulating that would make it seem very likely that diet can be considered an aetiological factor (Christakis et al., 1966; Turpeinen et al., 1968; Dayton et al., 1968). Even if included, it is impossible at the moment to assess the importance of dietary factors compared with other predisposing causes.

Much has been laid at the door of dietary fat as far as its importance in the causation of coronary thrombosis is concerned. It is some years now since it was established that the substitution of vegetable fat for animal fat in the diet resulted in a reduction of the level of serum cholesterol (Kinsell et al., 1952; Groen et al., 1952). It has been found that the fatty acids, active in reducing a raised serum cholesterol level are the polyunsaturated fatty acids linoleic acid (18:2) and arachidonic acid (20:4) (Ahrens et al., 1957). The evidence that animal fat, which is composed largely of saturated fats, is a potent factor in the causation of atheroma and coronary artery disease is not conclusive, but is nevertheless, sufficiently circumstantial for the American Heart Association in 1964 to issue a warning to the public that at least half of the fat intake of the U.S. citizen (approximately $40 \%$ of the calories) should be as unsaturated or vegetable fat and furthermore, that the total fat intake should be reduced. In 1968, the collective medical boards of the Scandinavian countries released a statement to the public which contained the following remarks: "cutting down on the total consumption of fat in combination with a reduction in the amount of saturated fats and a relative increase in the quantity of polyunsaturated fats can diminish the risk of atherosclerosis. The consumption of vegetables, fruit, potatoes, skimmed milk, fish, lean meat and cereal products should be increased.'

There seems little doubt that cholesterol in the diet can influence the level of cholesterol in the serum (Connor, Stone \& Hodges, 1964). However, this measure is hardly within the bounds of practical possibility. If the diet is cholesterol-free (a very uninspiring diet) then the addition of cholesterol will raise the level of serum cholesterol up to a daily consumption of approximately $300 \mathrm{mg} /$ day. Above this level of intake little further increase in the serum cholesterol occurs until the intake is over $5 \mathrm{~g}$ or so per day. When it is considered that one egg contains $250 \mathrm{mg}$ cholesterol, and to keep below $300 \mathrm{mg} /$ day only skimmed milk and small portions of meat can be taken with no cheese, butter or animal fat, then this is not a way of reducing serum cholesterol that will appeal to any but the most determined.

Dietary findings from the Harvard School have shown that some fatty acids are more likely to be associated with raising the serum cholesterol than others (Hegsted et al., 1965). The fatty acids concerned are myristic (14:0) and palmitic (16:0). If this work is confirmed, milk fat is going to come under fire.

What about carbohydrates in the diet and do they. have any part to play in the causation of coronary artery disease?

In addition to satisfying hunger, carbohydrates, as simple sugars, have the ability to satisfy appetite and a dietary survey in the U.S.A. has shown that though the proportion of carbohydrate eaten in that country is little changed compared with the beginning of the century the type of carbohydrate eaten has changed (Antar, Ohlson \& Hodges, 1964). Nowadays in the Western world much of the dietary carbohydrate eaten is in the form of simple di- or mono-saccharides rather than as the complex starches.

Not many years ago it was thought that all dietary carbohydrates were converted to glucose and therefore it did not matter what type was eaten. This is now known not to be so and in view of the association between serum lipids and coronary thrombosis it would be of interest to learn whether the lipid changes brought about by the modern tendency to consume more simple carbohydrates were in the direction of those associated with coronary disease. 
It has been suggested that the increased incidence of ischaemic heart disease is due to an increased sucrose intake (Yudkin, 1957) but the evidence for this, though supportive, is far from conclusive. We are certainly not in a position at this moment to say whether those metabolic effects of sucrose that are different from other dietary carbohydrates are advantageous or disadvantageous to man. In the past 10 years or so several groups of workers from all over the world have reported that, in man, different carbohydrates have different effects on lipid metabolism mainly as judged by the level of fasting serum triglycerides (see Macdonald, 1970). There is some conflicting evidence on the effect of the type of dietary carbohydrate on cholesterol metabolism and it is mainly the triglyceride metabolism that has been found to be sensitive to the nature of the dietary carbohydrate.

In those communities with a high incidence of coronary disease, carbohydrates are not usually consumed without fat, at approximately the same calorie level and it is possible that the combination of certain dietary fats with certain dietary carbohydrates may be more atherogenic than the sum of the two given alone. Conversely the combination of a certain carbohydrate with a certain fat may prevent any undesirable feature found when either substance is given alone. It has been found that the combination of sucrose with a polyunsaturated fat in the diet prevented the increase in fasting serum triglycerides that occurs when the sucrose is given either alone or with cream (Macdonald, 1967). The metabolic effects of interrelationships between the various foods we eat is relatively unexplored and could well have more consequences on our well-being than the lack of interest suggests.

Not only what we eat, but how we eat seems to be of some importance in determining the lipid and other responses to the constituents of the diet. Ischaemic heart disease is commoner among those who take three meals or less per day (Fabry et al., 1968).

Death from vascular disease is creeping down the age-scale, in this country to such an extent that the foundations of these disturbances are possibly laid in childhood. What then are the factors in childhood that accelerate this process?

Coronary intimal infiltration more than doubles in the male between the ages of 10 and 20 years (Holman et al., 1958) and this is correlated with maximal androgen-production and a corresponding appetite for rich foods such as eggs and butter (Dock, 1959).

Well-known are the necropsy findings in American service men killed in Korea. The average age of death was 22 years and $50 \%$ had grossly visible disease of the aorta, and in $10 \%$ the lumen of at least one coronary artery was reduced to less than half itš normal size. In contrast, no such deposits were found in the aortas of Chinese and Korean soldiers (Enos, Beyer \& Homes, 1955). The American men. were obviously healthy to be in the services. A coms? parable study in the U.S. Air Force on men at necropsy whose average age was 28 years showe $\underline{\underline{5}}$ that $70 \%$ had coronary artery disease (Glantz $8 \%$. Sternbridge, 1959). There is no reason to believe tha the U.S. findings do not apply to this country.

What then can be done about the diet in childhood in order to minimize the risks of coronary throm? bosis, especially in male children where the chances $\overrightarrow{-}$ of getting a blocked coronary before the age of $5 \omega^{\circ}$ years are much greater than in females?

No dietary advice can be said with certainty to be effective but it would be prudent to reduce the animat fat the child consumes, and possibly encourager the eating habit of 'nibbling' rather than 'gorging' $\omega$

Thus it seems that at the moment there is now absolutely conclusive evidence that dietary fats ob carbohydrates play a role in atherosclerosis and subsequent heart disease. The evidence that points in this direction is, however, strong and it woulक possibly be wise at this stage to accept the inferencê of this evidence and to give dietary advice accordsingly. Probably not all individuals are equally reace $\vec{\theta}$ tive to dietary constituents and to decide whether individual has a carbohydrate- or fat-induced typenof lipid abnormality would be a useful guide to a pre? ventive diet.

What dietary advice is to be given to a person whఖ for ono or more reasons is in this coronary-pron group? Obviously over-weight should be correcte -reduction in weight leads to a reduction of serun? lipid levels and thus to a reduction of the incidences of myocardial infarction. It seems from the know ledge we have at the moment that the fat intake should be reduced and its constituents should cons tain more polyunsaturated fatty acids. The daily intake of food should be taken little and often rather than at one major meal a day followed by a collapse into the nearest armchair. The findings, at the moment, certainly do not justify a mass campaign to reduce sucrose consumption in men and elderly women. Who knows, but if we eat a combination o重 foods such as vegetable oil with our sucrose an glucose with our cream, we may still be able t $\odot$ enjoy almost the best of all worlds?

\section{References}

Ahrens, E.H., Insull, W., Bloomstrand, R., Hirsch, Tsaltas, T.T. \& Peterson, M.L. (1957) The influence of dietary fats on serum lipid levels in man. Lancet, $i, 9430$ Antar, M.A., Ohlson, M.A. \& Hodges, R.E. (1964) Changes in retail market food supplies in the U.S. in the last seventy years in relation to the incidence of coronary heart disease with special reference to dietary carbo 
hydrates and essential fatty acids. American Journal of Clinical Nutrition, 14, 169.

Christakis, G., Rinzler, S.H., Archer, M., Winslow, G., Jampel, S., Stephenson, J., Friedman, G., Fein, H., Kraus, A. \& James, G. (1966) The anti-coronary club. American Journal of Public Health, 56, 299.

Connor, W.E., Stone, D.B. \& Hodges, R.E. (1964) Interrelated effects of dietary cholesterol and fat upon human serum lipid levels. Journal of Clinical Investigation, 43, 1691.

Dayton, S., Pearce, M.L., Goldman, H., Harnish, A., Plotkin, D., Shickman, M., Winfield, M., ZaGer, A. \& Dixon, W. (1968) Controlled trial of a diet high in unsaturated fat for prevention of atherosclerotic complications. Lancet, ii, 1060.

Dock, W. (1959) Why are men's coronary arteries so sclerotic? Journal of the American Medical Association, $170,152$.

Enos, W.F., Beyer, S.C. \& Holmes, R. (1955) Pathogenesis of coronary disease in American soldiers killed in Korea. Journal of the American Medical Association, 158, 912.

FABRY, P., Fodor, J., HeJl, Z., GeIZEROVA, H. \& BalCAROVA, O. (1968) Meal frequency and ischaemic heart disease. Lancet, ii, 190.

Glantz, W.M. \& Sternbridge, V. (1959) Coronary artery atherosclerosis as a factor in aircraft accident fatalities. Journal of Aviation Medicine, 302, 75.
Groen, J.J., Tijong, K.B., Willebrands, A.F. \& KaMMINGA, C. (1952) Influence of nutrition, individuality and different for ms of stress on the ser um cholesterol. Voeding, 13, 556.

Hegsted, D.M., McGandy, R.B., Myers, M.L. \& Stare, F.J. (1965) Quantitative effects of dietary fat on serum cholesterol in man. American Journal of Clinical Nutrition, $17,281$.

Holman, R.L., McGill, H.C., Strong, J.P. \& Geer, J.C. (1958) The natural history of atherosclerosis. American Journal of Pathology, 34, 209.

Kinsell, L.W., Partridge, J.W., Boling, L., Margen, S. \& Michaels, G.D. (1952) Dietary modification of serum cholesterol and phospholipid levels. Journal of Clinical Endocrinology and Metabolism, 12, 909.

MACDONALD, I. (1967) Interrelationship between the influences of dietary carbohydrates and fats on fasting serum lipids. American Journal of Clinical Nutrition, 20, 345.

MACDONALD, I. (1970) Effect of dietary glycerol on the serum glyce ide level of men and women. British Journal of Nutrition, 24, 537.

Turpeinen, O., Miettinen, M., Karovnen, M.J., Roine, P. Peknarinen, M., Lehtosuo, E.J. \& Alivirta, P. (1968) Dietary prevention of coronary heart disease. American Journal of Clinical Nutrtion, 21, 255.

YUDKIN, J. (1957) Diet and coronary thrombosis. Lancet, ii, 155. 